\title{
A modified split Hopkinson torsional bar in studying shear localization
}

\author{
Q Xue, L T Shen and Y L Bai \\ Laboratory for Nonlinear Mechanics of Continuous Media, Institute of Mechanics, \\ Chinese Academy of Sciences, Bejijing 100 080, People's Republic of China
}

Received 20 February 1995, in final form 23 May 1995, accepted for publication 27 June 1995

\begin{abstract}
A modified split Hopkinson torsional bar (SHTB) is introduced to eliminate the effect of the loading reverberation of the standard SHTB on the study of evolution of shear localization. The effect, the cause and the method by which to eliminate loading wave reverberation are carefully analysed and discussed. By means of the modified apparatus, the post-mortem observation of tested specimens can provide data on actual evolution of micro-structure and micro-damage during shear localization. Some test results of shear banding conducted with this apparatus support the use of the modified design. Moreover, the modification makes possible the correlation of evolving micro-structures to the transient shear stress-strain recording.
\end{abstract}

\section{Introduction}

The split Hopkinson torsion bar is one of the most commonly used instruments for the study of behaviour of materials under dynamic loading. Since Baker and Yew [1] first developed this apparatus, a number of investigators have improved this technique [2-7]. These modifications mainly focused on loading type, configuration of stress wave and change of strain rate bistory for the studies of dynamic response of materials. Because of the remarkable advantages of SHTB, for example, no effects of wave dispersion and friction, this technique has been widely used in studying shear localization phenomena $[8,9]$.

Some experimental studies were tecently carried out on the evolution of shear localization with SHTB $[10,11]$. For instance, Marchand and Duffy [10] investigated the process of macroscopic deformation by short-exposure photography and measured the temperature distribution across the localized zone. Although the experimental method adopted by Marchand and Duffy has shed light on the process of shear localization, their technique is unable to reveal the development of microstructure and micro-damage during shear localization.

In this paper, we present a new experimental method, the interrupted test with the SHTB technique, to investigate the evolution process of a shear band. The key point of this method lies in examining the micro-structure of tested specimens at any stage of shear banding through a post-mortem observation. Obviously, a single wave loading and an adjustable loading duration are required. However, the standard SHTB cannot meet that requirement because of the loading wave reverberation. For this reason, a modification of SHTB is introduced to eliminate the effect of loading reverberation and to provide a continuously adjustable loading duration for the planned study. A detailed stress wave analysis is given and the characteristics of loading waves before and after the modification are compared. The effect of the modified SHTB on loading strain rate is discussed. The observation of micro-structure of the shear band shows the effectiveness of this modification. The modified SHTB makes feasible the interrupted test and thus enables the correlation of evolution of microstructure to the transient shear stress-strain response.

\section{The principle of the modified torsional Hopkinson bar}

The shear localization under high strain rate undergoes a transient process from the uniform deformation to the development of a shear band in a local zone of the specimen. To capture the evolving characteristics of micro-structure during this dynamic process is no doubt very difficult. Here, a new experimental method, called the interrupted test, is proposed to investigate the evolution of shear band micro-structure. Its main conception is based on the view that the localized micro-structure at different loading stages can be 'frozen' on the specimens. A series of tests with identical specimens were performed on the SHTB with constant stored torque but different loading durations. By unloading at different positions on the nominal stress-strain curve, a group of specimens with different levels of residual plastic deformation can be gained. The post-mortem observation for these tested specimens can provide the information on the progress of shear localization. For example, the observations and measurements of the scribed lines that were deposited on the surface of the tubular specimen 
before the tests can provide the history of the deformation distribution during shear localization. Moreover, metallurgical observations of the same sites can reveal the changes in micro-structure and micro-damages during the process.

Obviously, a single torsional loading pulse with a controllable loading duration is required to correlate the transient stress-strain measurement to post-mortem observation of deformed micro-structure. In this respect, the standard SHTB cannot fulfil the aims of the planned study because of the reverberation of torsional waves resulting from wave reflections. The standard SHTB consists of two bars with a tubular specimen fixed between them. The released stress wave on the standard SHTB is reflected from both ends of the bar system and exerts subsequent multiple loading on the specimen. Figure 1 shows an oscillogram of a standard SHTB test with a dummy solid specimen. The trace presents the state of the torque wave recorded on the transmission bar. Several loading pulses of the same amplitude and duration follow the first one. Undoubtedly, if the amplitude of the secondary loading pulse exceeds the secondary yield strength of the specimen, the successive plastic deformation will occur, destroying the micro-structural pattern of shear localization produced by the first stress pulse. This problem is inherent to the structure of standard SHTB and has long been neglected when the micro-structure of a deformed specimen tested on the apparatus was examined. In order to remove the inherent disadvantage, the standard SHTB had to be modified to eliminate the effect of the loading reverberation on the micro-structure.

To investigate the effect of the reverberation of torsional waves in the SHTB, a simple stress wave analysis is made, based on the following assumptions.

(i) The specimen is treated as an interface because the gauge length is much less than the length of torsional bars.

(ii) At the interface, the partition of the incident wave into a reflection and a transmission wave is assumed to be a constant $K=M_{t} / M_{\mathrm{i}}$.

According to the one-dimensional stress wave theory,

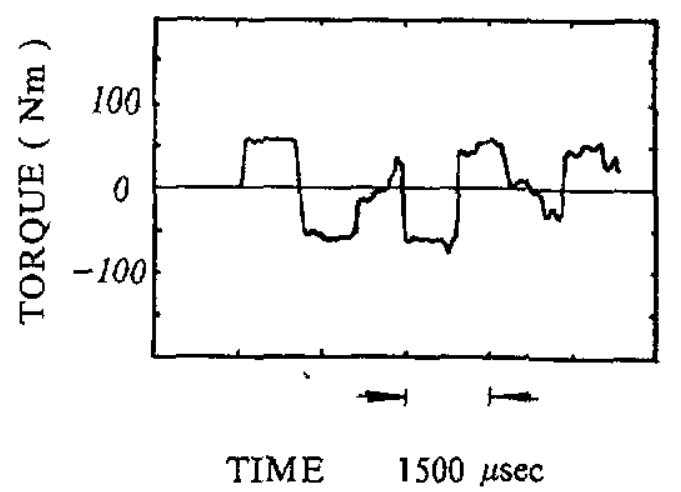

Figure 1. The oscillogram of a standard SHTB with a dummy specimen. the torsional wave equation is

$$
\frac{\partial^{2} \theta}{\partial t^{2}}=\frac{G}{\rho} \frac{\partial^{2} \theta}{\partial x^{2}}=C^{2} \frac{\partial^{2} \theta}{\partial x^{2}}
$$

where $\theta$ is the torsional angle in unit length and $x$ is the axial coordinate along the bar. $G$ and $\rho$ are the shear modulus and the density of the bars respectively. $C$ is the torsional wave speed. The characteristics of the waves are

$$
\tau \pm \rho c v=\text { constant along } x \pm c t=\text { constant }
$$

where $\tau$ and $v$ are the stress and the particle velocity of the sectional element adjacent to the outer surface of the bar.

In the analysis, the loading end of the incident bar is assumed to be fixed and the end of the transmission bar free. If we assume that we can neglect the effect of loading reverberation on the micro-structure in the first loading process, as most users of the standard SHTB did in practical tests before, then no further reflection of the stress wave would occur at the specimen interface after the first loading. For this case, the physical diagram $(x, t)$ and the wavestate $(\tau, v)$ are calculated and shown in figure 2. Here, $\tau_{0}$ and $v_{0}$ are given by

$$
\tau_{0}=\frac{1}{2} \frac{M r}{J_{b}} \quad v_{0}=\frac{\tau_{0}}{\rho c}
$$

where $M$ is the stored torque and $r$ and $J_{\mathrm{b}}$ are the radius and the moment of inertia of the bars, respectively.

According to the wave analysis, in zone 13 of figure 2 , the specimen will sustain a secondary reverse torque with the same amplitude as the first incident torque. A comparison of wavestates between the calculation (the full line) and the test recording (the broken line) at point $E$ is shown in figure 2 . The wave amplitude drop at $t^{*}$ just reveals the existence of additional wave reflection; therefore, a secondary plastic deformation must have occurred. Figure 3 is the oscillogram recorded at gauge $\mathrm{F}$ and the loading reverberations of a standard SHTB test are clearly displayed. In order to study the correlation of the post-mortem observation of deformed specimen to the transient nominal stress-strain curve, the effect of the loading reverberation in the SHTB must be eliminated.

The primary source of the loading reverberation in the SHTB is attributed to the residual energy retained in the bar system. Hence, developing a method by which to transfer the loading wave out of the SHTB system after the first loading is necessary in order to reduce loading reverberations.

The stress wave analysis of the SHTB indicates that the loading pulse is divided into reflected and transmitted waves when it meets the specimen, as shown in figure 2 . The reflections of these waves at the ends of the two bars result in the secondary loading on the specimen. It is difficult to lead the reflected wave out of the incident bar due to the complexity of structure at the loading end. An expedient measure is to limit the effect of reflected wave from the incident bar on the secondary loading. By controlling the amplitude of the loading 


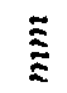

$\stackrel{5}{5}$

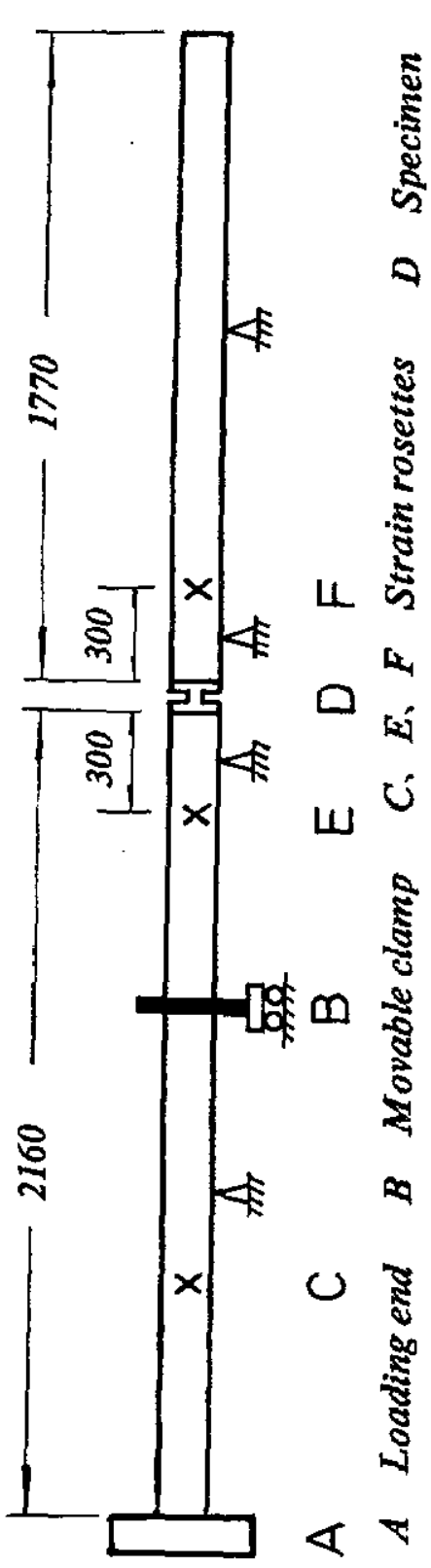

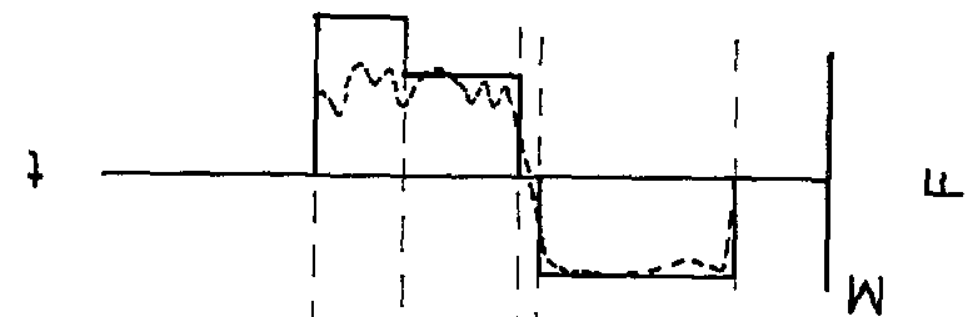

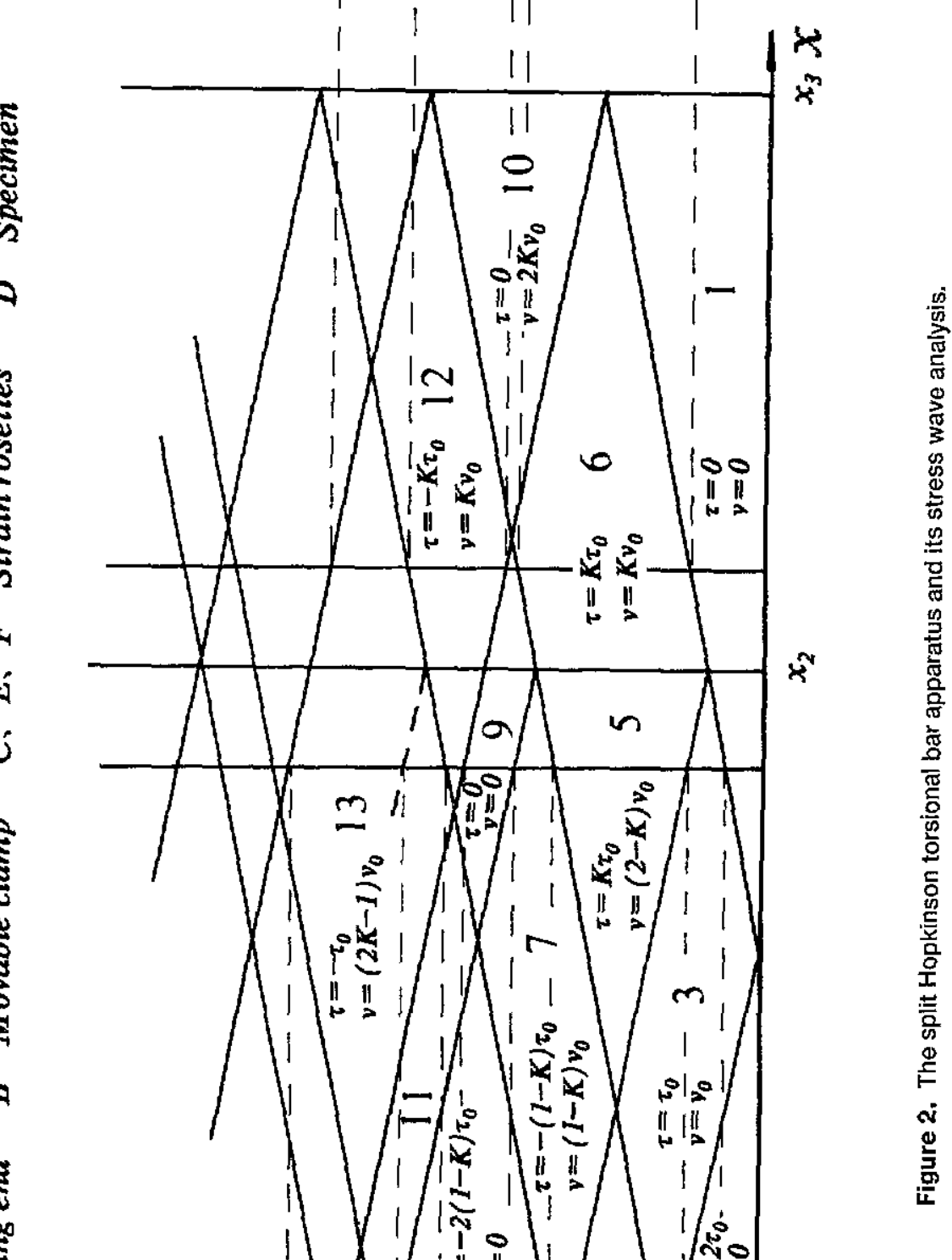

7

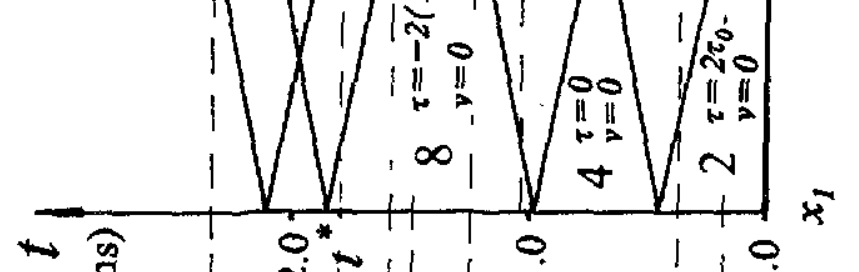

山 


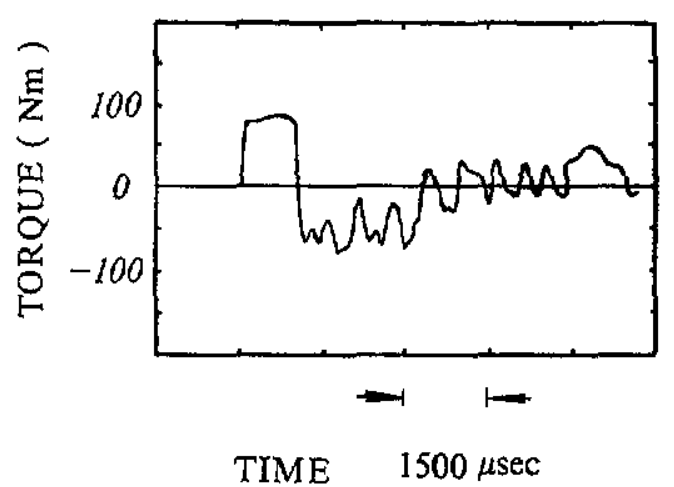

Figure 3. The oscillogram of a test on a standard SHTB at the gage F. Several subsequent loading pulses following the first one acted on the specimen.

pulse, the amplitude of the reflected wave in the incident bar can be reduced to a level that only causes elastic response in the specimen. At the same time, the sizes of specimen are properly adjusted to ensure minimum decrease of loading strain rate due to the control of wave amplitude. However, the transmitted wave with sufficient amplitude to reach the strength limit of the tested material needs to be led out of the bar system. Thus, the modified design ensured that the tested materials will not sustain the secondary plastic deformation.

\section{The modification of the SHTB}

To eliminate the reflection of a transmitted wave at the free end of the transmission bar, an additional bar, namely the unloading bar, was attached to the transmission bar (figure 4). If the unloading bar is first fixed with the transmission bar to admit wave propagation from the latter to the former without wave reflection, and then can be separated after the transmitted wave passes into the unloading bar completely and before the front of its reflected wave comes back to the interface $x_{3}$ between them, then no reflected torque would return to the transmission bar from the unloading bar. This requires

$$
L \geqslant L_{1}-x_{\mathrm{m}}
$$

where $L$ and $L_{1}$ are the lengths of the unloading bar and the incident bar respectively and $x_{\mathrm{m}}$ is the minimum distance between the clamp and the specimen.

In the above conception, two key points have to be satisfied: the connection of the two bars for wave propagation and the separation of the two bars at the right moment for wave isolation. According to the stress wave analysis (figure 4), when the transmitted wave reflected at the free end of the unloading bar reaches $x_{3}$, the unloading bar will rotate faster than the transmission bar. Using this feature, a clutch that will only conduct the torsional wave unilaterally and comes apart automatically due to the difference in rotational speeds between transmission bar and unloading bar (figure 5) was developed. The clutch consists of two collars (marked as $\mathrm{A}$ and $\mathrm{B}$ ) that are glued to the transmission and unloading bars, respectively. Initially the clutch is fixed as shown in figure 5(a). Four sliders (marked C) put in the guide of collar A are inserted into collar B. If the rotational angular speed $\omega_{\mathrm{A}}$ is equal to or greater than $\omega_{\mathrm{B}}$, then the torsional wave will propagate from collar A to collar B through sliders $C$ (see figure $5(a)$ ). Once $\omega_{\mathrm{A}}$ has become smaller than $\omega_{\mathrm{B}}$, the sliders $C$ will be pushed back into the guide of collar $A$ by the slope guide of collar B (see figure $5(b)$ ). The separation between the transmission and unloading bars is automatically completed. Thus, the transmission wave is segregated from the bar system. This clutch, called the insidepushed clutch due to its operating principle, is shown in figure 6 . The unloading bar associated with the insidepusbed clutch eliminates the reflection of the transmission wave. Under the condition of controlling wave loading, the modified technique makes it possible to investigate the evolution of shear band micro-structure.

For the current SHTB, the length of the incident bar and the minimum distance between the clamp and the specimen are 2160 and $500 \mathrm{~mm}$, respectively. According to the analysis above, the length of the unloading bar should exceed $1660 \mathrm{~mm}$. Made of Ly12 aluminium alloy (similar to $2024 \mathrm{Al}$ ), as were the other bars, a $1780 \mathrm{~mm}$ long bar of $25 \mathrm{~mm}$ outside diameter was selected as the unloading bar. Figure 7 gives an oscillogram trace of dynamic calibration of the modified SHTB with a dummy specimen. As anticipated, only one loading pulse is observed except for several subsequent small peaks with about a third or a half of the amplitude of the transmitted wave and about $200 \mu$ s duration. They may be attributed to the wave reflection at the interface of the clutch due to its imperfect contact. Figure 8 shows an oscillogram of a test with the transmission rate $K=0.6$. Obviously, the secondary loading pulse has about a third the amplitude of the transmitted pulse. The modification effectively removes the reflection of the transmitted wave. It needs to be pointed out that the subsequent waves after the first loading pulse are not in good agreement with the predicted one. This effect can be explained by the fact that the assumption of the fixed end at $x_{1}$, when the reflected wave reaches the interface, does not hold perfectly, because of the change in rotating direction of the incident bar, during which the shaft key between the incident bar and the great inertia loading system cannot retain complete contact in both rotating directions. During this period, the end condition is approximately free to make a normal wave in some of zone 12 in figure 4 . The lower the reflected wave, the longer the effect lasts. Of course, the clutch's reflection also exerts its effect on this process, but its relatively short duration and lower amplitude (shown in figure 7) make it unable to result in disagreement over such a long period.

\section{Discussion of the modified SHTB}

The modified SHTB eliminated the effect of secondary loading on the plastic deformation of specimens, but it 

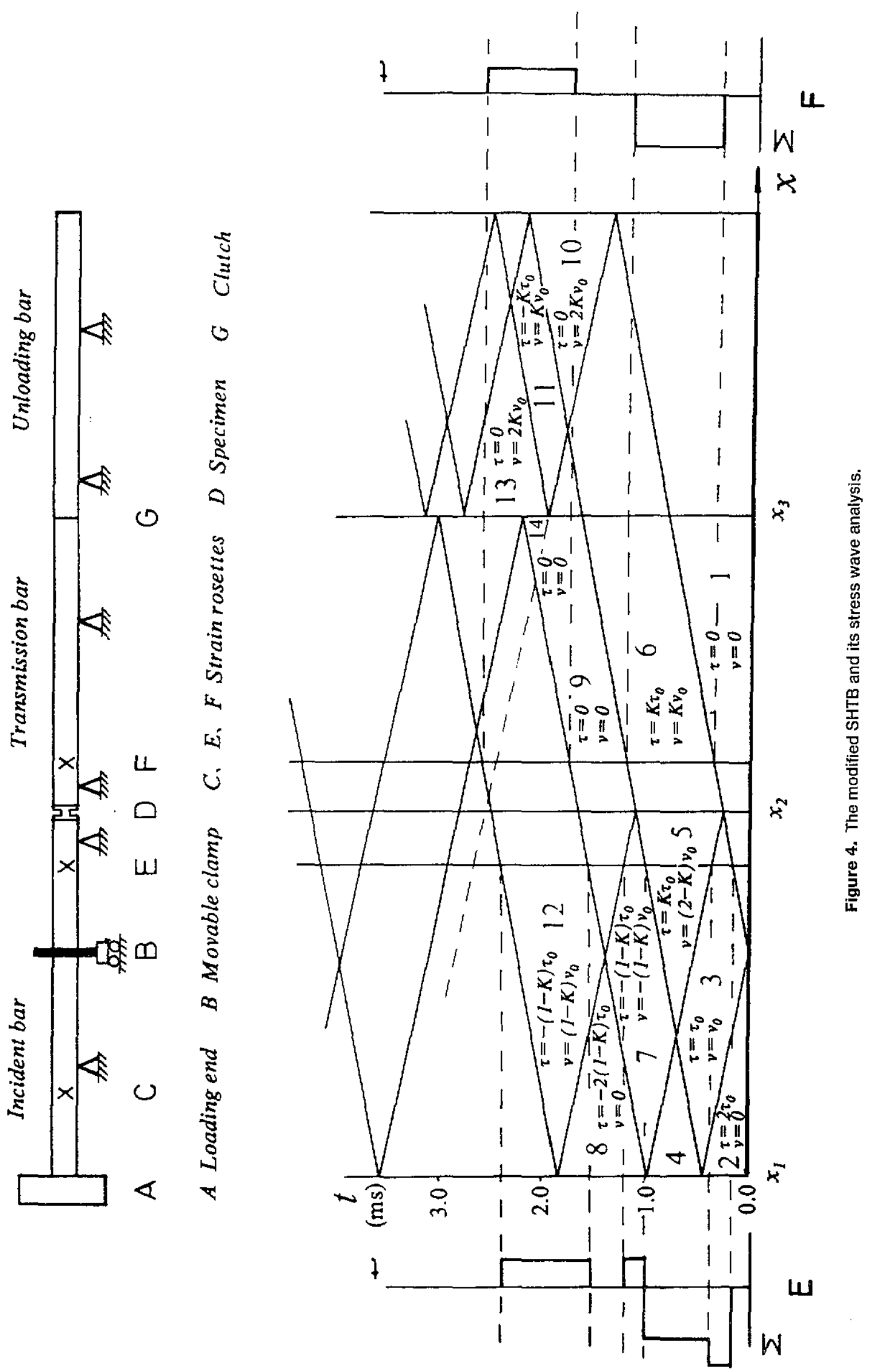


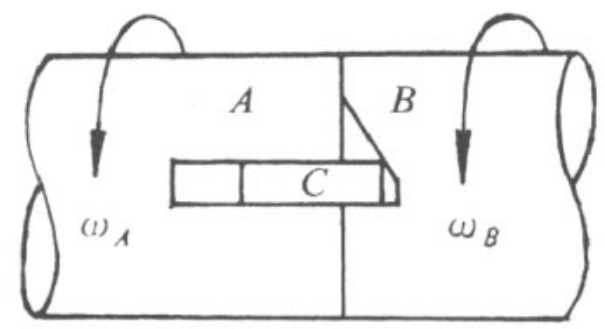

(1) ${ }_{A} \geqslant \omega_{B}$

(a)

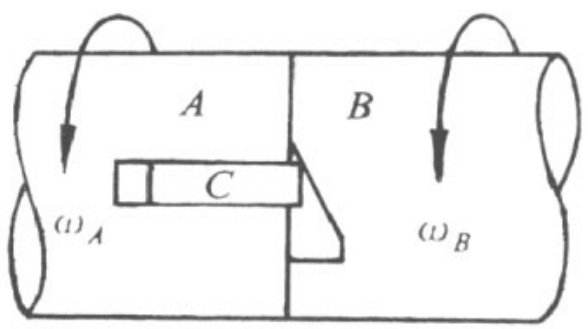

$\left(\omega_{1}<{ }^{(1)_{B}}\right.$

(b)

Figure 5. The principle of the inside-pushed clutch.

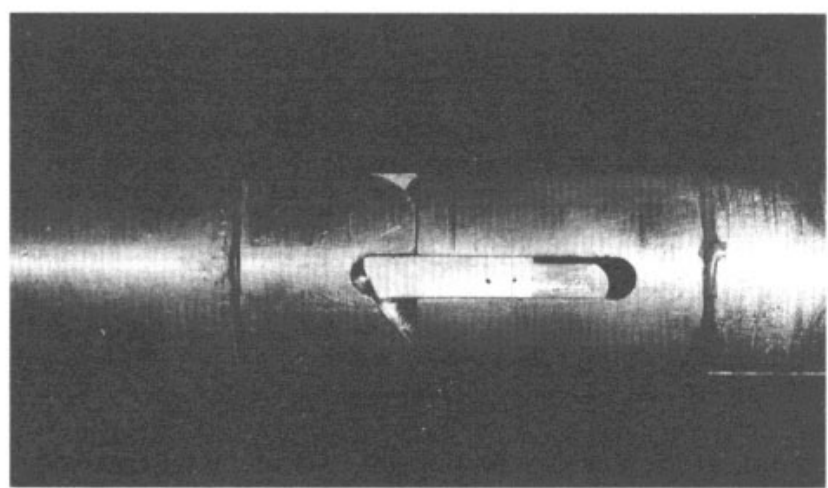

Figure 6. The inside-pushed clutch.

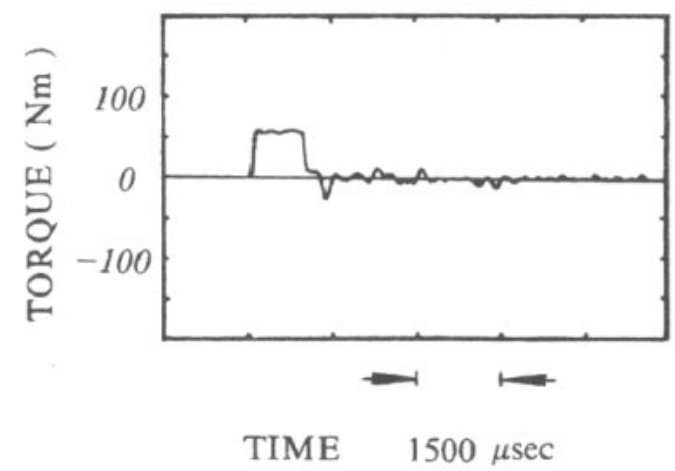

Figure 7. The oscillogram of the modified SHTB with a dummy solid specimen.

could limit the amplitude of the initial loading wave and, therefore, the loading strain rate. The following discussion offers an estimation of this effect.

From the principle of the SHTB, the average strain rate is given by

$$
\dot{\gamma}=\frac{r_{\mathrm{s}} C}{l_{\mathrm{s}} J_{\mathrm{h}} G}\left(M_{\mathrm{i}}-M_{\mathrm{r}}-M_{\mathrm{t}}\right)
$$

where $M_{\mathrm{i}}, M_{\mathrm{r}}$ and $M_{\mathrm{t}}$ are the torques of loading, reflected

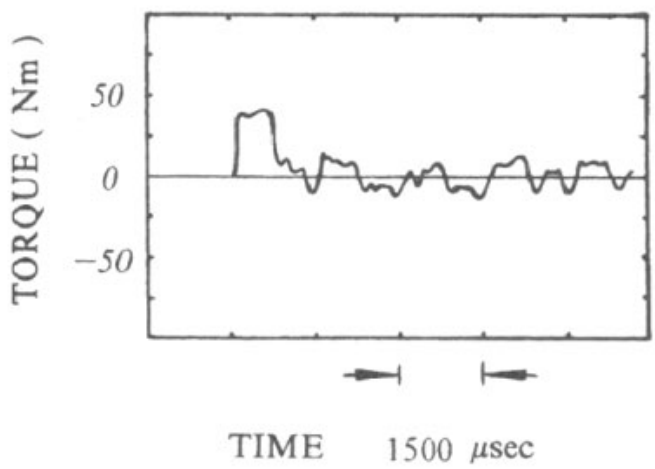

Figure 8. The oscillogram of a test on the modified SHTB at the gage $F$.

and transmitted waves respectively and $r_{\mathrm{s}}$ and $l_{\mathrm{s}}$ are the equivalent radius and the gauge length of the specimen. Consider the condition of the loading wave:

$$
\begin{gathered}
M_{\mathrm{i}}+M_{\mathrm{r}}=M_{\mathrm{t}} \\
M_{\mathrm{t}}=J_{\mathrm{s}} \tau_{\mathrm{b}} / r_{\mathrm{s}}
\end{gathered}
$$

where $\tau_{\mathrm{b}}$ is the shear strength of the specimen and $J_{5}$ is the moment of inertia of the thin-walled specimen. On combining equations (6) and (7), equation (5) becomes

$$
\dot{\gamma}=\frac{2 r_{\mathrm{s}} C}{l_{\mathrm{s}} J_{\mathrm{b}} G}\left(M_{\mathrm{i}}-\frac{\tau_{\mathrm{b}} J_{\mathrm{s}}}{r_{\mathrm{s}}}\right)=\dot{\gamma}_{\mathrm{s}}\left(\frac{M_{\mathrm{i}}}{M_{\mathrm{t}}}-1\right)
$$

where the characteristic strain rate is

$$
\dot{\gamma}_{\mathrm{s}}=\frac{2 J_{\mathrm{a}} \tau_{\mathrm{b}} C}{l_{\mathrm{s}} J_{\mathrm{b}} G} .
$$

The modified SHTB limits the amplitude of the incident torque from $M_{\mathrm{i}}$ to $M_{\mathrm{ig}}$. The torque relation becomes $M_{\mathrm{ig}}+M_{\mathrm{rg}}=M_{\mathrm{tg}}$ (the subscript $\mathrm{g}$ denotes the variables relative to the modified SHTB). Obviously, 
$\left|M_{\mathrm{rg}}\right|$ is the amplitude of the secondary incident torque.

It is required that

$$
\begin{gathered}
-M_{\mathrm{rg}} \leqslant J_{\mathrm{s}} \tau_{\mathrm{s}} / r_{\mathrm{s}} \\
M_{\mathrm{ig}} \leqslant \frac{J_{\mathrm{s}} \tau_{\mathrm{s}}}{r_{\mathrm{s}}}+M_{\mathrm{gg}}
\end{gathered}
$$

where $\tau_{s}$ is the reverse secondary yield stress of the specimen. Considering the case of equality in equation (10), the loading strain rate of the modified SHTB will be

$$
\dot{\gamma}_{\mathrm{g}}=\frac{2 r_{\mathrm{s}} C}{l_{\mathrm{s}} J_{\mathrm{b}} G}\left(M_{\mathrm{ig}}-M_{\mathrm{tg}}\right)=\frac{2 r_{\mathrm{s}} C}{l_{\mathrm{s}} J_{\mathrm{b}} G} \frac{\tau_{\mathrm{s}} J_{\mathrm{s}}}{r_{\mathrm{s}}}=\dot{\gamma}_{\mathrm{s}} \frac{\tau_{\mathrm{s}}}{\tau_{\mathrm{b}}}=\dot{\gamma}_{\mathrm{s}} \alpha
$$

where $\alpha=\tau_{\mathrm{s}} / \tau_{\mathrm{b}}$ represents Bauschinger's effect. Thus, we have

$$
\frac{\dot{\gamma}_{g}}{\dot{\gamma}}=\frac{\alpha}{\left(M_{\mathrm{i}} / M_{\mathrm{t}}\right)-1} .
$$

Generally, the ratio $M_{\mathrm{i}} / M_{\mathrm{t}}<3$ for a well-matched design of a real test. For ideal plastic or isotropic hardening materials $(\alpha=1)$, the limitation of the loading wave to the modified SHTB decreases the loading strain rate by a factor of two relative to the standard SHTB. To take account of a possible Bauschinger effect and the thermal softening effect during the late stage of shear localization, a more conservative estimation about the limitation of loading strain rate needs to be given. If $\alpha$ is selected to be 0.5 , then the loading strain rate of the modified SHTB will be decreased by a factor of four from the unmodified value. This analysis indicates that the modified SHTB is able to ensure a loading strain rate of the same order as that of the standard one.

\section{A case study of shear localization}

An important application of the modified SHTB is to study the process of shear localization; in particular, the intrinsic relationship between a transient shear stressstrain curve and the evolution of micro-structures during shear localization. A set of tests was performed with the unmodified and the modified SHTB. A comparison of the test results justified the effectiveness of the modified apparatus for the planned objective.

A group of tests of a hot-rolled steel sample was carried out on the standard SHTB. The micro-structure of the deformed specimen is shown in figure 9. In the undeformed specimen, the ferrite grains and pearlite colonies arranged along the rolled direction were perpendicular to the shear direction. Ordinarily, the prcipitated lines of pearlite should have been distorted along the shear direction. However, these lines in the centre of the shear zone have been reversely distorted, after the first shear loading, to their original undeformed positions. Some of them even evolved a reverse slope and constituted a local reverse 'S' pattern (marked by arrows in figure 9). Undoubtedly, the reverse loading

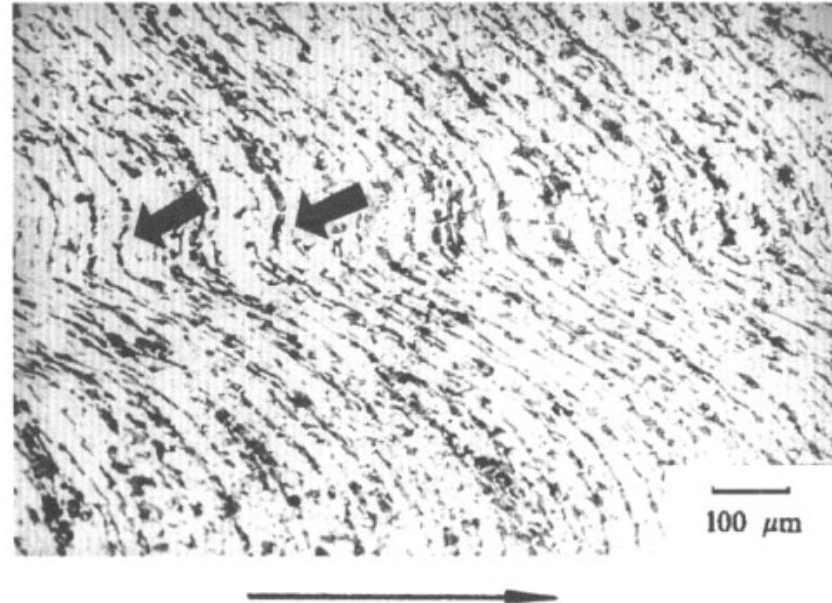

Figure 9. A destroyed micro-structure of shear localization for a hot-rolled steel sample due to the reverberation of the loading wave of a standard SHTB (the arrows mark the reverse 'S' kink.

wave in the standard SHTB made the specimen sustain the secondary plastic deformation and destroyed the micro-structure of shear localization. In fact, the residual image of micro-structure resulted from the multiple loading process due to the loading reverberation of the standard SHTB.

As a comparison, another group of tests of a tempered hot-rolled steel sample were conducted with the modified SHTB to examine the micro-structural characteristics of a shear band. From the principle of the modified SHTB, the amplitude of the incident wave was selected to be about twice that of the transmitted wave. This will remove the secondary plastic deformation imposed on the specimen, if Bauschinger's effect is not very severe. The micrograph shown in figure 10 displays the distinct structure of shear localization. Although there is a slight hardening effect in the steel, the microstructure shows no trace of reverse plastic deformation (see figure 10).

Furthermore, a group of tests of Ti-6Al-4V alloy were carried out to investigate the process of shear localization with the interrupted test method [12]. In these tests the amplitude of the loading wave was limited to about 1.6 times that of the transmitted wave. The wave oscillogram showed no subsequent loading wave of amplitude more than a third that of the incident wave, and thus the tested specimens were verified as being loaded without the secondary plastic deformation. Figure 11 shows the correlation of the transient $\tau-\gamma$ curve and corresponding micro-structure and micro-damage in the shear band during the course of localization. At four different stages of the process of shear banding, the micro-structure of deformed specimens changed from 


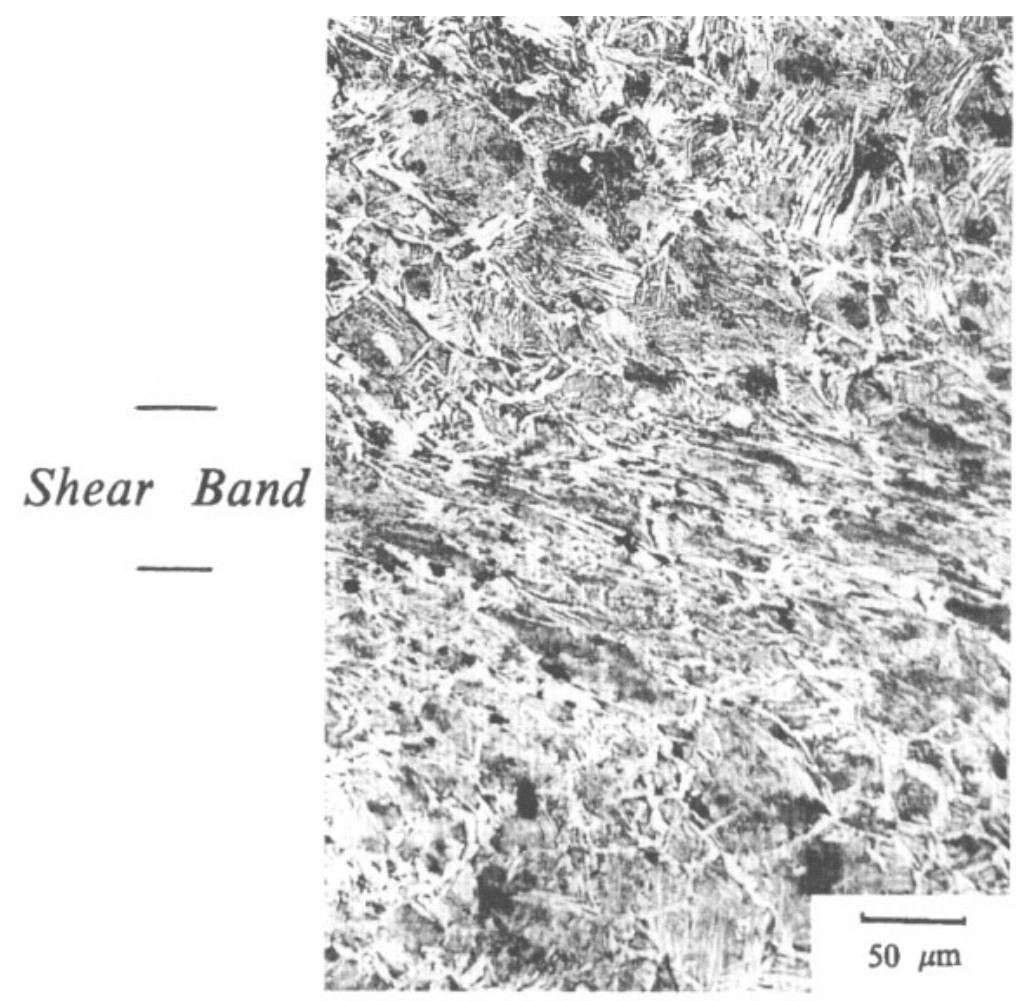

Figure 10. A pattern of the shear band micro-structure of a deformed specimen loaded by the modified SHTB.

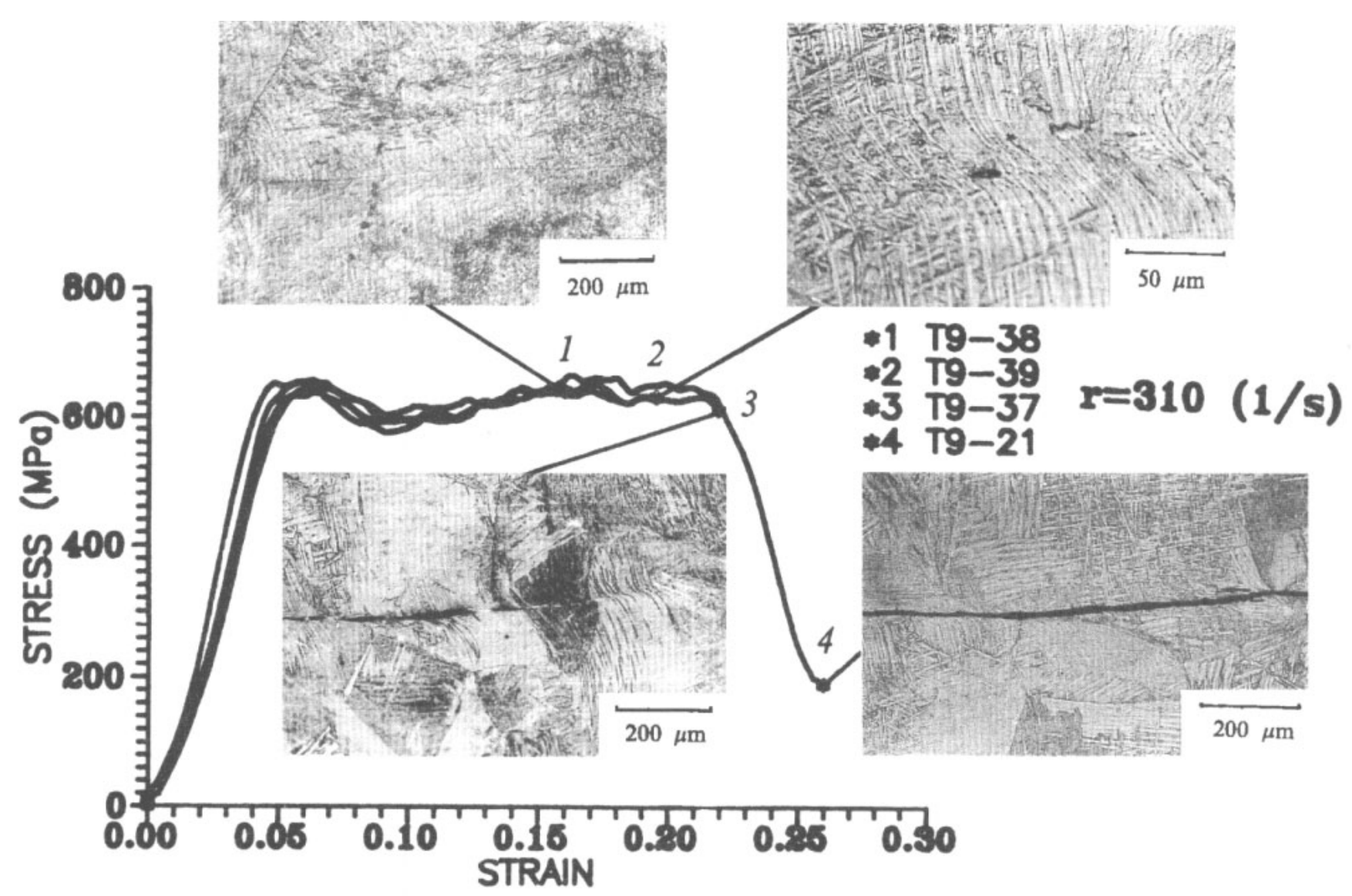

Figure 11. The correlation between transient $\tau-\gamma$ curves and corresponding micro-structure during the process of shear localization in Ti-6Al-4V alloy. 
unstable (point 1) to failure (point 4). The photographs with different scales help to clarify the details of the progressive accumulation of microvoids during shear localization.

\section{Summary}

A new experimental method was proposed in order to investigate the evolution of micro-structure in shear localization. A modified SHTB was introduced to eliminate the effect of loading reverberation and made possible the quantitative analysis of localized deformation through post-mortem observation of tested specimens. The key to the modified SHTB lies in an additional unloading bar and an automatically separated clutch. If the incident torque is chosen properly, then the modified SHTB can ensure that no secondary plastic deformation of a specimen can occur after the first torsional wave pulse. With the modified SHTB, the interrupted test method becomes a feasible way to investigate the evolution of micro-structure during the process of shear localization. A detailed discussion indicates that the limit of the incident torque will not have a significant effect on the loading strain rate so long as the Bauschinger effect of the tested material is not very severe. The experimental oscillograms and the metallurgical observations justify the modification of the SHTB.

\section{Acknowledgment}

This work was supported by the Chinese National Natural Science Foundation.

\section{References}

[1] Baker W E and Yew C H 1966 Strain-rate effects in the propagation of torsional plastic wave J. Appl. Mech. Trans. ASME E 33 917-23

[2] Campbell J D and Dowling A R 1970 The behaviour of materials subjected to dynamic incremental shear loading $J$. Mech. Phys. Solids 18 43-63

[3] Nicholas T 1971 Strain-rate and strain-rate history effects in several metals in torsion Exp. Mech. 11 370-4

[4] Duffy J, Campbell J D and Hawley R H 1971 On the use of a torsional split Hopkinson bar to study rate effects in 1100-0 aluminum J. Appl. Mech. Trans. ASME E 38 83-9

[5] Frantz R A and Duffy J 1972 The dynamic stress-strain behavior in torsion of $1100-0$ aluminium subjected to a sharp increase in strain rate J. Appl. Mech. Trans. ASME E 39 939-45

[6] Lipkin J, Campbell J D and Swearengen J D 1978 The effects of strain-rate variations on the flow stress of OFHC copper J. Mech. Phys. Solids 26 251-68

[7] Gilat A and Pao Y H 1988 High-rate decremental-strainrate test Exp. Mech. 28 322-5

[8] Costin L S, Crisman E E, Hawley R H and Duffy J 1979 On the localization of plastic flow in mild steel tubes under dynamic torsional loading Proc. 2nd Conf. on Mechanical Properties at High Rates of Strain ed J Harding (Bristol: IOP Publishing) pp 90-100

[9] Hartley K A, Duffy J and Hawley R H 1987 Measurement of the temperature profile during shear band formation in steels deforming at high strain rates J. Mech. Phys. Solids 35 283-307

[10] Marchand A and Duffy J 1988 An experimental study of the formation process of adiabatic shear bands in a structural steel J. Mech. Phys. Solids 36 251-83

[11] Giovanola J H 1988 Adiabatic shear banding under pure shear loading-part I: direct observation of strain localization and energy dissipation measurements Mech. Mater. $759-71$

[12] Xue Q 1991 An experimental study of evolution of thermo-visco-plastic shear localization in Ti-6Al-4V alloy and No. 20 steel Thesis Institute of Mechanics, Academia Sinica 